\title{
RoboTAP: Target priorities for robotic microlensing observations
}

\author{
M. Hundertmark ${ }^{1}$, R. A. Street ${ }^{2}$, Y. Tsapras ${ }^{1}$, E. Bachelet ${ }^{2}$, M. Dominik ${ }^{3}$, K. Horne ${ }^{3}$, V. Bozza ${ }^{4}, 5$, D. M. Bramich ${ }^{18}$, \\ A. Cassan ${ }^{6}$, G. D’Ago ${ }^{7}$, R. Figuera Jaimes ${ }^{3,8}$, N. Kains ${ }^{8,9}$, C. Ranc ${ }^{10}$, R. W. Schmidt ${ }^{1}$, C. Snodgrass ${ }^{11}$,

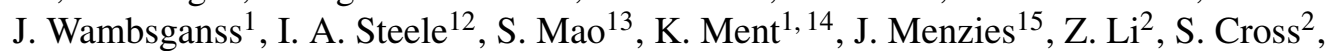 \\ D. Maoz ${ }^{16}$, and Y. Shvartzvald ${ }^{16,17}$
}

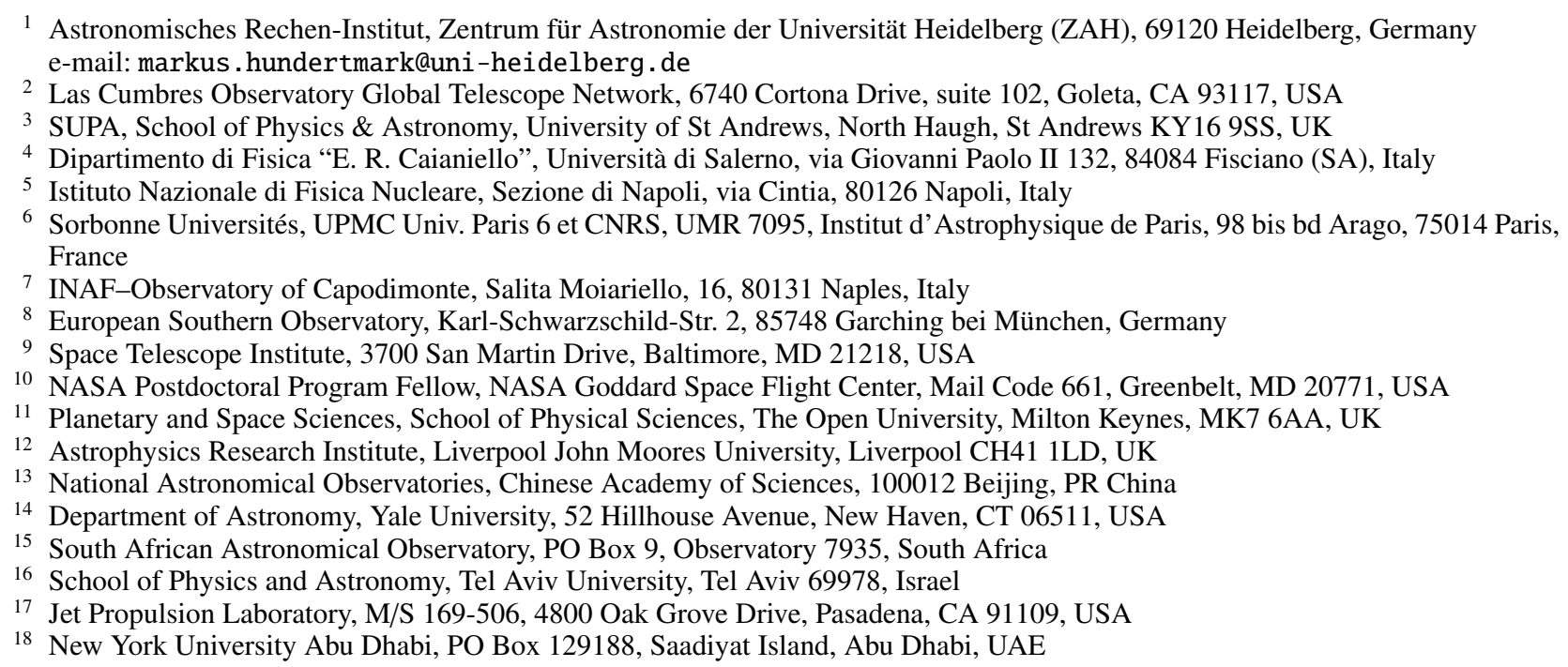

Received 24 February 2017 / Accepted 28 September 2017

\begin{abstract}
Context. The ability to automatically select scientifically-important transient events from an alert stream of many such events, and to conduct follow-up observations in response, will become increasingly important in astronomy. With wide-angle time domain surveys pushing to fainter limiting magnitudes, the capability to follow-up on transient alerts far exceeds our follow-up telescope resources, and effective target prioritization becomes essential. The RoboNet-II microlensing program is a pathfinder project, which has developed an automated target selection process (RoboTAP) for gravitational microlensing events, which are observed in real time using the Las Cumbres Observatory telescope network.

Aims. Follow-up telescopes typically have a much smaller field of view compared to surveys, therefore the most promising microlensing events must be automatically selected at any given time from an annual sample exceeding 2000 events. The main challenge is to select between events with a high planet detection sensitivity, with the aim of detecting many planets and characterizing planetary anomalies.

Methods. Our target selection algorithm is a hybrid system based on estimates of the planet detection zones around a microlens. It follows automatic anomaly alerts and respects the expected survey coverage of specific events.

Results. We introduce the RoboTAP algorithm, whose purpose is to select and prioritize microlensing events with high sensitivity to planetary companions. In this work, we determine the planet sensitivity of the RoboNet follow-up program and provide a working example of how a broker can be designed for a real-life transient science program conducting follow-up observations in response to alerts; we explore the issues that will confront similar programs being developed for the Large Synoptic Survey Telescope (LSST) and other time domain surveys.
\end{abstract}

Key words. gravitational lensing: micro - methods: observational - methods: statistical

\section{Introduction}

\subsection{RoboTAP in context}

In an era of increasing sky coverage and telescope étendue, surveys monitor billions of stars and alert us whenever an astronomical object changes its brightness. Most science cases will require follow-up observations responding to these alerts. This is especially true for transient events that, by definition, occur only once. The sheer number of alerts issued by the Large Synoptic Survey Telescope (LSST), for example, can be on the order of one million per night (Ridgway et al. 2014) and therefore cannot be handled by humans. Brokers for processing and filtering the alert stream for rapid response telescopes already exist, for instance the Arizona-NOAO Temporal Analysis and Response to Events System (ANTARES; Saha et al. 2016), but more work is needed to address the variety of science cases. The demand 
for such systems will increase due to emerging projects such as the Evryscope (Law et al. 2015), the Zwicky Transient Facility (Smith et al. 2014; Bellm 2014), the BlackGEM survey and last but not least the LSST (LSST Science Collaboration et al. 2009). The RoboTAP system is just such an automated broker system and a prototypical example for a range of transient science cases.

The philosophy behind the RoboTAP implementation is to provide a robust and computationally lightweight algorithm that only takes seconds for an event feed with $\sim 1000$ events. In order to achieve that, we follow a small set of basic working principles. We start by carrying out computationally inexpensive operations first and apply increasingly complex criteria later; we replace estimates that are only required to be roughly known by suitable interpolants. Incoming event parameters are kept and updated in memory all the time. The same technical considerations apply to selecting a suitable and algebraically simple priority function.

Figure 1 depicts the simplified workflow of RoboTAP acting as a broker between the event stream and the observation manager. The main sources for the alert stream are existing microlensing surveys such as the Optical Gravitational Lensing Experiment (OGLE) survey (Udalski et al. 1994) or the Microlensing Observations in Astrophysics (MOA) survey (Bond et al. 2004). The workflow illustrates how an incoming stream of events is subjected to filters and triggers observations that in turn can refine the event parameters in a closed-loop system. It also highlights that further building blocks are necessary to efficiently process the event stream. Only events that have been classified as microlensing are processed. Moreover, we introduce two sub-classes - regular and anomalous microlensing events. For that purpose, we rely on the Automated Robotic Terrestrial Exoplanet Microlensing Search (ARTEMiS) system (Dominik et al. 2008) providing us with event parameters and anomaly triggers for events with irregular light curve shapes. In this two-tier approach, we generate preliminary target lists by reducing the number of transient (microlensing) events based on observability, survey coverage, and expected duration. Finally, we rank all events based on our priority function. Anomalous and non-anomalous events are allocated a pre-defined fraction of the observing time on the network. The corresponding target lists are then formatted as separate observing requests and submitted to the telescope network.

\subsection{Related observatories}

While our approach is of general interest for all astronomical observatories, the actual implementation was customized to the Las Cumbres Observatory. The Las Cumbres Observatory (LCO) is an organization carrying out astronomical research and operating a global network consisting of homogeneous telescopes of different aperture classes (Brown et al. 2013). In addition to the two $2 \mathrm{~m}$ Faulkes telescopes originally used by the RoboNet collaboration (Burgdorf et al. 2007; Snodgrass et al. 2008; Bramich 2008; Tsapras et al. 2009; Street et al. 2011), $9 \times 1 \mathrm{~m}$ telescopes became available in 2013 to the microlensing program, of which eight are on sites in the southern hemisphere, where most of the microlensing targets lie. The geographical location of telescopes as shown in Fig. 2, ensures that targets can be observed nearly seamlessly if weather permits. The lower density of observations above Australia is a consequence of having only $2 \times 1 \mathrm{~m}$ telescopes available, as well as the characteristic weather pattern.

With an emphasis on robotic time-domain astronomy, gravitational microlensing perfectly fits into the observing program

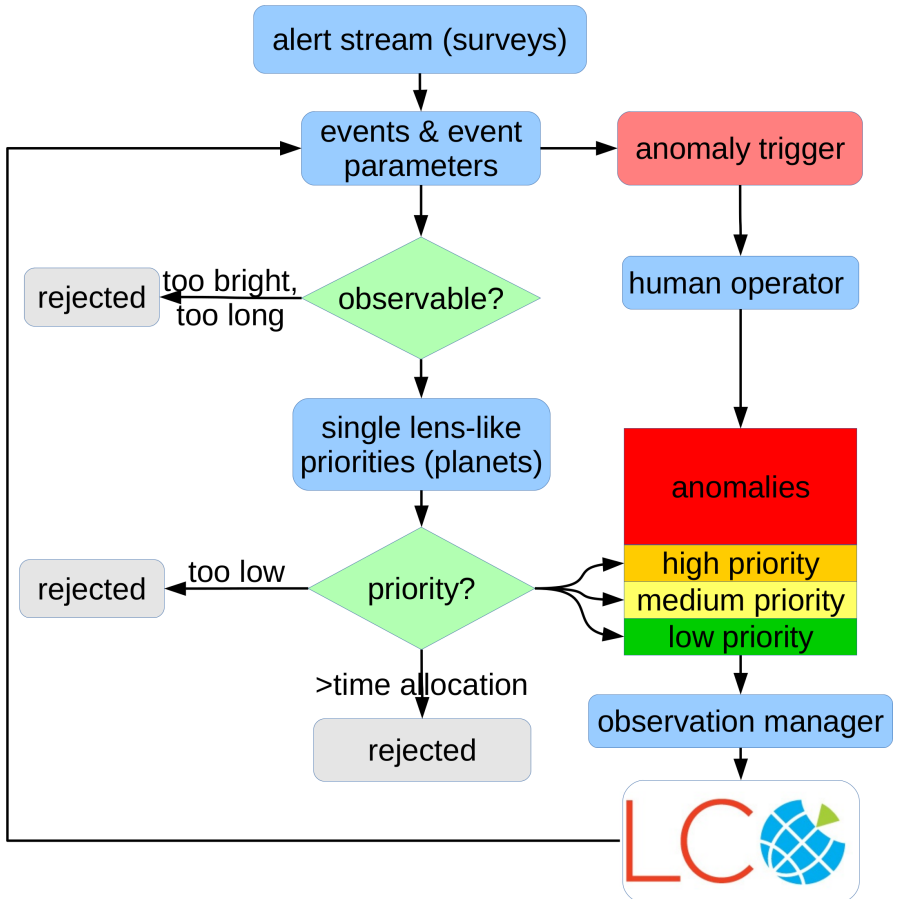

Fig. 1. Workflow of the RoboTAP system processing the incoming alert stream of regular and anomalous microlensing events from ARTEMiS.

of LCO. Moreover, with its telescope clusters in Chile, South Africa, and Australia, it achieves unprecedented coverage of events in the Galactic center. Consequently, microlensing was selected to be part of the 2013 pilot program of the $1 \mathrm{~m}$ network and was awarded Key Project ${ }^{1}$ status on the LCO network between 2014-2016. At the beginning of the 2013 pilot phase, the multi-purpose scheduler was not available and the telescopes were initially equipped with Santa Barbara Instrument Group (SBIG) model STZ-16803 cameras ${ }^{2}$. The Key Project was interrupted by the 2015 Spitzer campaign as described by Yee et al. (2015) for obtaining simultaneous ground and space-based observations of microlensing events. This paper will focus on the pilot phase and the first year of operations with its lower number of competing projects, direct scheduling, and corresponding camera systems.

\subsection{Gravitational microlensing}

Gravitational microlensing is one of the well-established methods of exoplanet detection, probing the Galactic planet population down to Earth-mass range with ground-based observations (Bennett \& Rhie 1996; Wambsganss 1997). Light from a distant source star is gravitationally deflected by a foreground lens star, producing an increase of brightness of the source from the point of view of the observer. Planets orbiting faint (even unseen) lens stars can be discovered in this manner, because the light curve shape is related to the mass of the lens-system and depends on the mass ratio between planet and host star as well as their angular separation. Moreover, the planet population beyond the snowline can be probed due to the increased sensitivity of this detection technique (Park et al. 2006). This is the main science driver of the aforementioned Key Project.

\footnotetext{
1 PI: R. A. Street.

2 http://LCO.net/network/instrumentation/ 1m-sbig-camera
} 


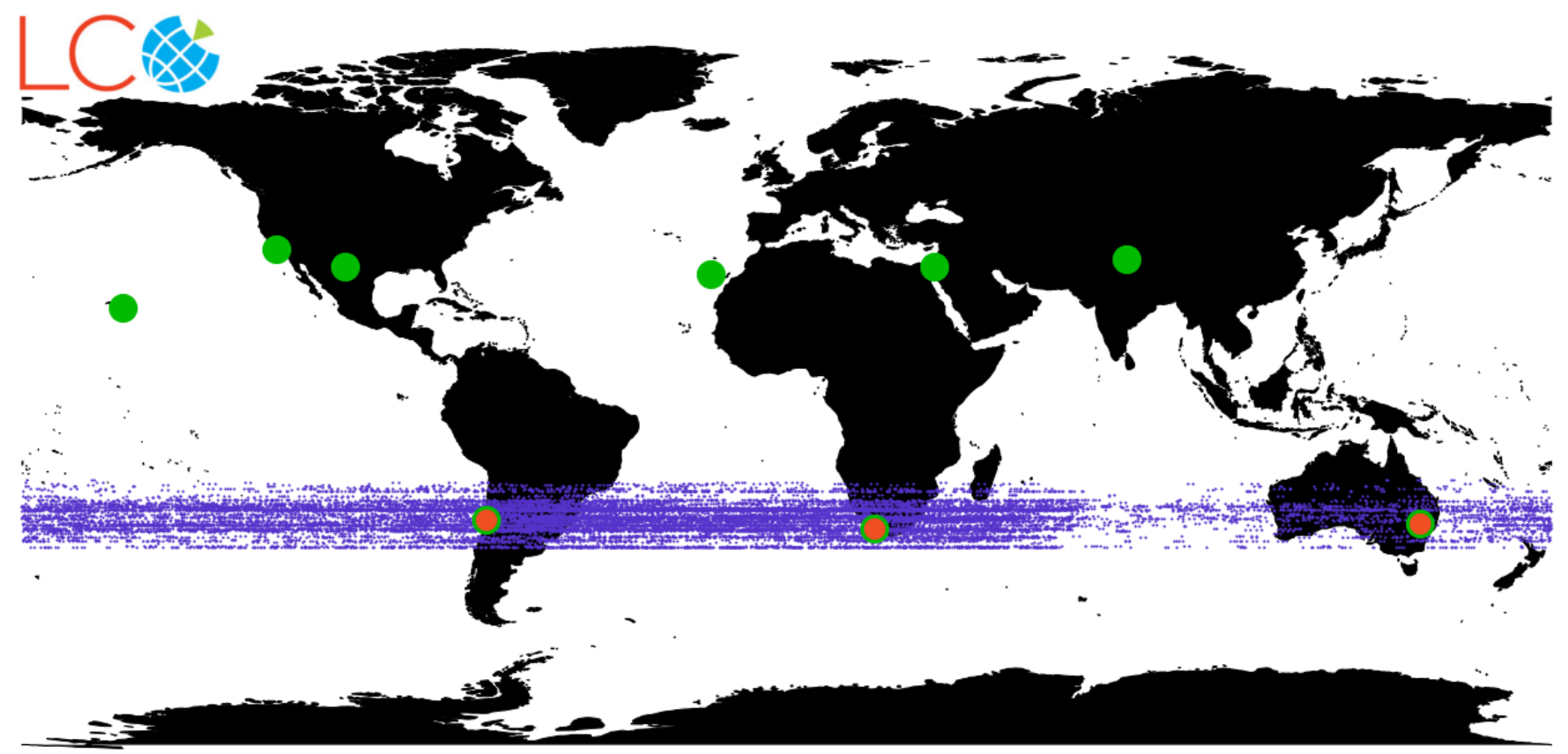

Fig. 2. Sites hosting LCO telescopes (green) and LCO sites used for the Key Project (red) are shown along with observed microlensing target positions at zenith over Earth for the 2015 season (blue dots). The Australian site hosts only two telescopes, which leads to less coverage, otherwise the event distribution would be homogeneous along all longitudes.

The sample of microlensing events and their absolute sensitivity to planets provides a statistical and independent measure of the planet abundance in our Galaxy (Snodgrass et al. 2004; Gaudi et al. 2002; Gould et al. 2010; Sumi 2010; Sumi et al. 2011; Cassan et al. 2012). The detection of microlensing events itself is subject to statistics, as only approximately one in a million stars in the Galactic bulge is sufficiently aligned with a deflecting foreground star as to ensure that more light will be redirected to the observer, as predicted by the seminal paper of Paczynski (1986). Both planet detections and non-detections provide important constraints on the frequency and distribution of planets in the Galaxy.

Most microlensing events follow a characteristic symmetric light curve, which is a direct consequence of Einstein's deflection angle. This deflection can be described by a simple lens equation, relating source position, image position, and deflection angle. The solutions to the lens equation provide the image positions. The radius of the ring-like image in a co-linear lenssource-observer configuration defines the Einstein radius

$\theta_{\mathrm{E}}=\sqrt{\frac{4 G M_{\mathrm{L}}}{c^{2}}\left(D_{\mathrm{L}}^{-1}-D_{\mathrm{S}}^{-1}\right)}$,

constraining the typical angular scale of the effect, where $D_{\mathrm{L}}$ denotes the distance from observer to deflecting lens of mass $M_{\mathrm{L}}$, and $D_{\mathrm{S}}$ denotes the distance to the source star. The total observable brightness increase is time dependent due to the relative source-lens proper motion. While multiple images can be detected and resolved for massive, extra-galactic source-lens configurations, microlensing on Galactic scales means that the image separation is of the order of $\sim 1$ mas and remains unresolved in the optical regime with current imaging technology. The total flux $F(t)$ of a microlensing event with a contribution of source flux $F_{\mathrm{S}}$ and blend flux $F_{\mathrm{B}}$ can be written as

$F(t)=F_{\mathrm{S}} A(u(t))+F_{\mathrm{B}}$, where the magnification $A$ of a single lens is obtained by adding the differential magnifications of each image $A_{ \pm}$, which depends on the actual image positions in units of the angular Einstein radius

$u_{ \pm}=\frac{u \pm\left(u^{2}+4\right)^{1 / 2}}{2}$.

The image magnification for the minor image $A_{-}$and the major image $A_{+}$is

$A_{ \pm}=\frac{u_{ \pm}^{2}}{u_{+}^{2}-u_{-}^{2}}$.

For practical purposes, we use the blend ratio

$g=\frac{F_{\mathrm{B}}}{F_{\mathrm{S}}}$,

and express the lens position in units of the angular Einstein radius. The time-dependent source-lens distance denoted as $u=u(t)$ defines the shape of the point-source point-lens (PSPL) Paczyński light curve

$A(u)=A_{+}+A_{-}=\frac{u^{2}+2}{u \sqrt{u^{2}+4},}$

in units of the angular Einstein radius. The latter expression can be derived analytically, because the lens equation of a single object can be solved for the image positions. The relative lens-source proper motion plays a role for the light curve shape, but for events that do not exhibit parallax effect (Gould 1992) a uniform motion along a straight line serves as a reasonable approximation on timescales of several days or weeks with the source-lens distance

$u=\sqrt{u_{0}^{2}+\left(\frac{t-t_{0}}{t_{\mathrm{E}}}\right)^{2}}$. 
The time of maximum magnification is denoted as $t_{0}$, the Einstein-radius crossing time is $t_{\mathrm{E}}$, and the minimum separation of lens and source is $u_{0}$. As an analytic model for all further considerations, Eq. (6) plays a crucial role for prioritizing events and detecting anomalies. In this context, we consider each event as anomalous if it deviates from the standard PSPL model as defined in Eq. (6) and is triggered by the anomaly detector algorithm described by Dominik et al. (2007). In a typical scenario, more than five clearly deviating points from at least two consecutive nights are required to confirm an anomaly.

The science driver of the LCO Key Project "Exploring Cool Planets Beyond the Snowline" is to find more planets with orbits between 0.5 and $10 \mathrm{AU}$, which corresponds to the orbital region where the temperature drops below the freezing point of water. Gravitational microlensing provides us with mass measurements and the projected orbital radii, thus populating the mass versus orbital radius diagram. Microlensing is complementary to the transit and the radial-velocity methods, since it is sensitive to Earth-mass planets beyond the water snowline.

\section{Prioritizing microlensing events}

\subsection{Priority and planet detection zones}

Microlensing follow-up programs can be adapted to different scientific objectives such as studying the brown-dwarf population (Street et al. 2013), providing mass measurements of distant stellar remnants (Mao et al. 2002; Wyrzykowski et al. 2016), or searching for planets beyond the habitable zone (Tsapras et al. 2014; Shvartzvald \& Maoz 2012). Searching for planets beyond the snowline is the main motivation behind the RoboNet-II program for following up microlensing events with telescopes from the Las Cumbres Observatory Global Telescope Network (LCO) and the Liverpool Telescope (Brown et al. 2013; Steele et al. 2004).

For the pilot phase of the $1 \mathrm{~m}$ network in 2013, we combined the approach of prioritizing events based on planet probability estimates with network specific observational constraints (Horne et al. 2009; Dominik et al. 2010). This approach addresses the challenge of selecting the right targets out of hundreds of ongoing events. For reasons of simplicity, one often considers an event to be ongoing when the separation between lens and source is smaller than the Einstein radius and the source thus experiences a brightening by at least 0.3 mag with respect to baseline. In practice, we permit observations for source-lens distances $|u|<1.5$ when an unblended source appears to be $0.1 \mathrm{mag}$ brighter. Figure 3 illustrates the number of available events at any time during the microlensing season (April to October). These 200-600 events include 5-10\% anomalous events deviating from a simple static point lens and are reported by anomaly detectors. Examples of different anomaly types can be found in Tsapras et al. (2016). Not all anomalous events can be monitored continuously, and even with two-to-three follow-up telescopes per site we can only cover a handful of events with high enough cadence. A suitable prioritization is therefore required.

The idea of defining the planet detection probability of a given microlensing event via its detection zone goes back to the initial suggestions of how likely it is that planets orbiting microlenses can be found (Gould \& Loeb 1992). A single-lens microlensing event creates a pair of images propagating through different parts of the lens plane where they can be perturbed by the presence of a planet. Estimating when such a perturbation becomes photometrically detectable gives the chance of finding

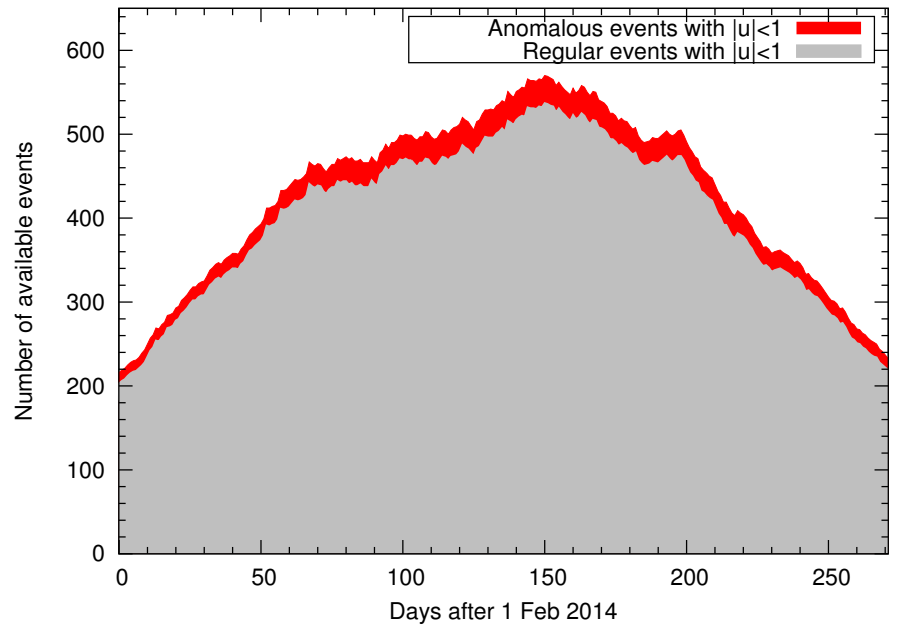

Fig. 3. Number of ongoing regular and anomalous events in the season 2014 before and after $t_{0}$.

such a planet. Figure 4 illustrates the locations where the extra planetary deflection is powerful enough to generate detectable deviations. The detection requires comparison with an underlying single-lens model, but the detection zone can shrink or grow depending on photometric accuracy. Therefore, one more commonly uses a $\Delta \chi^{2}$ threshold. For reporting a discovery one would require that observations lead to a change in $\Delta \chi^{2}>25$, corresponding to $5 \sigma$ detections, if the underlying uncertainties are appropriately assigned and follow a Gaussian distribution. The complicated way in which uncertainties are affected by crowded field photometry (Bachelet et al. 2015) frequently leads to a distribution of residuals that differs from that assumption and needs rescaling in the form of

$\sigma_{\text {rescaled }}^{2}=k\left(\sigma_{0}^{2}+\sigma_{\text {reported }}^{2}\right)$,

where $\sigma_{\text {reported }}$ refers to the original uncertainties and the parameters $k, \sigma_{0}$ are chosen so that the reduced $\chi^{2}$ becomes one for each independent light curve. Since some light curves have uncertainties that can be off by a factor of 2 , one commonly requires a threshold of $\Delta \chi^{2}>100$ for a detection.

In order to quickly calculate the planet detection probability $\Psi$, the exposure time $t_{\text {exp }}$ is adjusted according to the expected brightness. We thereby ensure that a photometric accuracy of 1.5 to $5 \%$ is reached and a planetary perturbation is unlikely to be missed. One of the main changes between the empirical relations by Horne et al. (2009) and Dominik et al. (2010), which are both based on the planet detection zone area, is the underlying logarithmic distribution of planets along the orbital axis, which is also consistent with observations (Cassan et al. 2012). The planet probability $\Psi$ for major and minor image positions $u_{ \pm}$ and their corresponding magnification $A_{ \pm}$is given by:

$\Psi(u)=\frac{2 A_{+}-1}{u_{+}^{\gamma}}+\frac{2 A_{-}}{u_{-}^{\gamma}}$,

where the parameter $\gamma$ is chosen to be two for the assumed population of planets, which comes from the aforementioned empirical relations combined with the assumption of a logarithmically distributed orbital axis. For $\gamma=2$ the detection probability can be simplified:

$\Psi(u)=\frac{4}{u \sqrt{u^{2}+4}}-\frac{2}{u^{2}+2+u \sqrt{u^{2}+4}}$. 

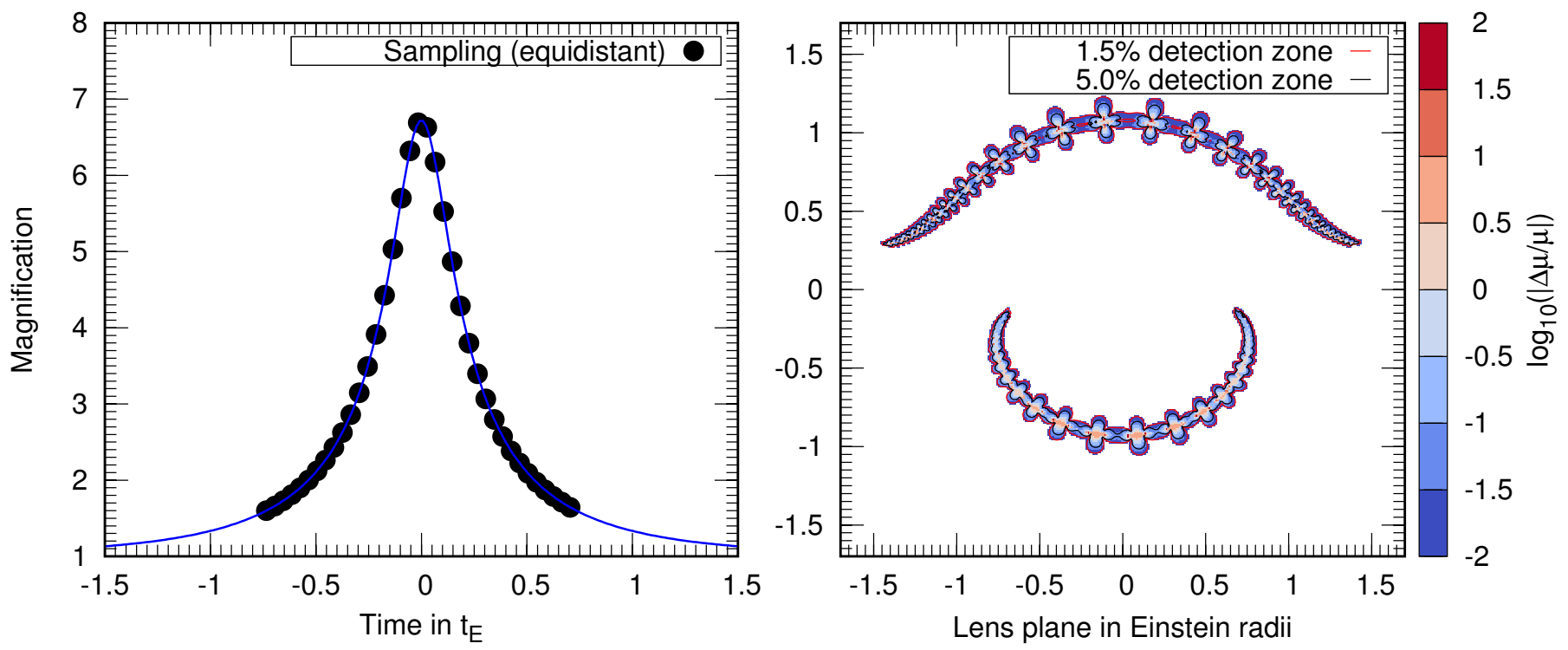

Fig. 4. Left: light curve of a standard point-lens light curve with a given equidistant sampling rate of one visit per night is shown. Right: corresponding planet detection sensitivity of planets perturbing the light curve is shown for the lens plane assuming a low-mass planet with mass ratio $q=10^{-4}$.

We consider the gain of observing an event to be given by the ratio of the planet detection probability and the invested observing time for a photon-noise dominated light curve. The priority function then reads

$\Omega_{\mathrm{S}} \propto \frac{A^{3 / 2} \Psi}{\left(A+F_{\mathrm{B}} / F_{\mathrm{S}}\right)\left(1+F_{\mathrm{B}} / F_{\mathrm{S}}\right)}$.

The latter expression makes implicitly the assumption that the suggested exposure time can be reached, that the noise model is correct, and that the underlying magnification $A$ is sufficiently constrained by fitting a PSPL model to the data. In the next section we will discuss to what extent these assumptions affect our results. One of the strategic differences between RoboNet and the strategy implemented in ARTEMiS is the absence of spending observing time on checking events when they slightly deviate from a point-source point-lens model. Based on estimates of earlier seasons, we expect that the priority function alone will deliver and select high priority events that eventually will turn out to be anomalous, including those of a potential planetary nature. Because of the two-step multi-tier strategy, it is more difficult to evaluate the sensitivity of our follow-up program. In order to ensure that anomalous events do not completely dominate our Key program, we have put a cap on the fraction of allocated anomalies.

To get a better understanding of where microlensing can find planets, we illustrate the detection zone in the right panel of Fig. 4. For each data point a single point lens creates two images sweeping along the Einstein ring. Depending on the proximity of these images to a potential planet, the light curve can be perturbed. For planets at various positions the corresponding deviation can be recorded and if it exceeds a certain threshold a planetary anomaly can be detected. The threshold depends on the achievable photometric accuracy as well as the desired number of deviating points. We assume that planets deviating by more than $1.5 \%$ (0.015 mag) can be detected if the exposure time can be adjusted accordingly.

For typical host stars ranging from 0.1 to $0.5 M_{\odot}$, we simulate the detection zone area for a mass ratio of $q \approx 10^{-4}$. This corresponds to planets in the range of three to 17 Earth masses covering super-Earths and mini-Neptunes. For typical point-lens parameters $\left(u_{0}=0.15, t_{\mathrm{E}}=25 \mathrm{~d}, \rho=10^{-3} \theta_{\mathrm{E}}\right)$ the $1.5 \%$ detection zone area shown in Fig. 4 also accounts for overlapping detection zones covering the same region. Finite source effects have been considered in the form of a uniform disk using the binary lens model by Bozza (2010) ${ }^{3}$. Most light curves can be simulated under the assumption that the source star is an infinitesimally small box and the distortion of that box is given by the inverse determinant of the lens equation. Whenever an image approaches a so-called critical line, the change of magnification is so dramatic that the surface of the source star is differentially magnified, which also ensures that the predicted brightness change remains finite.

Dominik (2009) hints at the unpredictability of events or in other words the difficulty of predicting the light curve days before the event reaches its maximum magnification. To successfully prioritize events, we expect that order relations

$\Omega_{\mathrm{S}, i}>\Omega_{\mathrm{S}, j}$

hold for each pair of consecutive events $i, j$. A heuristic estimate can be achieved assuming that the uncertainty of priority is limited by the uncertainty of $u$ as Eq. (11) monotonically increases with $u$. If the peak has not been reached and the blend flux is low, it is safe to assume that $u_{0} \in\left[0, F_{\text {now }} / F_{\mathrm{S}}\right]$. The corresponding uniform distribution and its variance $\Delta u / 12$ can be used to estimate the uncertainty in $u$ and for the last reported flux $F_{\text {now }}$ that provides an upper bound for $u$ that gives the uncertainty

$\sigma_{u}^{2} \approx \frac{F_{\text {now }}^{2}}{12 F_{\mathrm{S}}^{2}}$

If multiple events are considered before reaching their peak, those with the highest magnification estimate have automatically the lowest uncertainty and as long as the available events are separated by a factor of $1 / \sqrt{12}$ in $u_{0}$ the priority order is appropriate and that corresponds to the aforementioned variance of a

\footnotetext{
3 http://www.fisica.unisa.it/gravitationAstrophysics/ VBBinaryLensing.htm
} 
uniform distribution. For events with $t>t_{0}, u$ is much better constrained. After observing the peak on a single occasion with a relative accuracy of $\delta_{F}=1.5 \%$ and by estimating that $A \approx 1 / u$ and $A_{\text {true }}>A_{\text {peak, }}$

$\sigma_{u}^{2}<\delta_{F}^{2} u^{2}$

This can be interpreted by comparison with Eq. (13) for $F_{\text {now }} \approx$ $F_{\text {peak }}$, where the uncertainty $\sigma_{u}$ roughly follows $1 / u$ before the peak and $u$ after the peak. Before the peak is characterized, it is difficult to optimize the exposure time regardless of our knowledge of $g$. After the peak, a higher underlying signal-to-noise ratio in flux units is required where events with $g \gg 10$ may end up with more than $1 \%$ noise in $A$.

\subsection{Simulating a fiducial season}

Before analyzing the outcome of real observations, we shall provide an estimate of the expected number of events and achievable number of planets based on a simple model of basic microlensing parameters. For that purpose, we simulate ensembles of events with event parameters $t_{\mathrm{E}}, u_{0}, I_{\text {baseline }}, g$, where samples are drawn using a Gaussian kernel density estimate of PSPL parameters obtained from post-season ARTEMiS fits of the 2012 season. We will show at a later point that the real-time ranking is sufficiently close to the post-season estimate. For each event we simulate a random time in the season and let the system determine its current priority state. Based on that simulation we determine the priority thresholds for the season. The cumulative distribution of the priority function as shown in Fig. 5 indicates that $1 \%, 2 \%$, and $1 \%$ of events would fall in our low, medium, and high priority categories, respectively. Consequently, we would expect to request observations of 20,40 , and 20 non-anomalous events assuming that 2000 events are provided by survey teams.

In practice, the assumed number of underlying events turned out to be too low, because the revised simulation based on 2013 season parameters hints at an excess of low-priority targets. One explanation for the corresponding offset is that a new discovery channel of the Optical Gravitational Lensing Experiment (OGLE-IV) in its fourth phase (Udalski et al. 2015) is reporting fainter microlensing events, which has led to an increase of $15 \%$ in terms of discovered events; so effectively we should expect to see 40, 70, and 30 events within our thresholds. Fainter source stars that are magnified beyond the limiting magnitude are by definition of higher magnification and thus more likely to contribute to events with higher priorities.

Based on the confirmed planets in the exoplanet archive ${ }^{4}$, we have seen six planetary events with seven planets in the OGLE fields for 2012 and about 1700 stellar microlensing events in total. One event contains two planets (Han et al. 2013). With no assumption on the optimal choice for selecting events, we have a $1 / 5$ chance of seeing a single planet when covering 50 events. Our choice of parameters and detection probability $\psi$ can be calculated at the peak of each light curve, which is an approximation of the planet detection sensitivity of each event. That gives us a relative number of how many more planets we expect to see.

Our strategy automatically incorporates that approach by assigning a priority level $\Omega_{\mathrm{S}}$. The planets shown are based on the parameter records and not on published values to give us an indication of whether the system would have picked the target. While $99.9 \%$ of the peak planet detection probability is concentrated in 100 targets, two thirds of published planets instead belong to

\footnotetext{
4 http://exoplanetarchive.ipac.caltech.edu
}

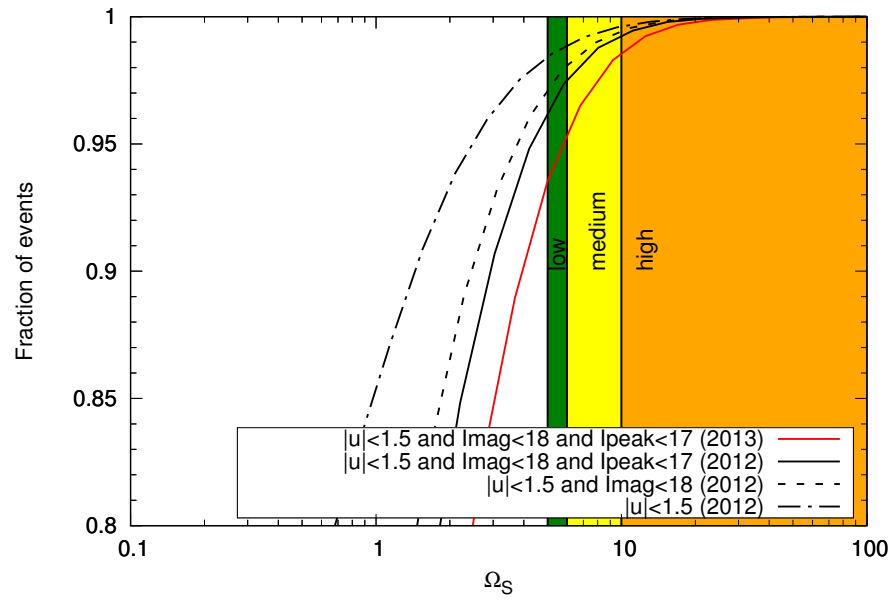

Fig. 5. Cumulative priorities based on simulated events matching the season 2012 are shown for the underlying observing constraints and after the season 2013 has finished.

the 200 highest priority targets. We have limited that estimate to data from 2013 onwards, because older data would misrepresent the input from the anomaly detection and available survey data. Planets in the wings can still be detected although the planet detection probability is several orders of magnitude lower if it is sampled in a way that prevents overlapping detection zone areas for many events.

\subsection{Characterizing events}

The most important physical parameter that is accessible through gravitational microlensing is the planet mass:

$M_{\mathrm{p}}=\frac{\mu t_{\mathrm{E}}}{\kappa \pi_{\mathrm{E}}} \frac{q}{1+q} ; \kappa \approx 8.14 \frac{\mathrm{mas}}{M_{\odot}}$,

where $\mu$ denotes the lens-source relative proper motion, $\pi_{\mathrm{E}}$ is the magnitude of the microlensing parallax vector expressed in units of the Einstein radius, $q=M_{\mathrm{p}} / M_{\text {host }}$ is the mass ratio, and $\kappa$ is a constant (Gould 2000). Propagating the uncertainties and writing all uncertainty contributions relative to their quantities yields:

$\sigma_{M_{\mathrm{p}}} \approx \frac{\mu q t_{\mathrm{E}}}{\kappa \pi_{\mathrm{E}}(1+q)}\left(\frac{\sigma_{t_{\mathrm{E}}}^{2}}{t_{\mathrm{E}}^{2}}+\frac{\sigma_{\pi_{\mathrm{E}}}^{2}}{\pi_{\mathrm{E}}^{2}}+\frac{\sigma_{\mu}^{2}}{\mu^{2}}+\frac{\sigma_{q}^{2}}{q^{2}(1+q)}\right)^{1 / 2}$.

For small-mass ratios, $1 /(1+q)$ is sufficiently close to one and thus the planet mass uncertainty is dominated by the term with the largest relative uncertainty. In the following, we will focus on the uncertainties of mass ratio and timescale since these can be constrained better for most events. In order to quantify how the uncertainties in the fit-parameters can be reduced, we use the total information content expressed in the Fisher matrix:

$I_{i, j}(t)=\left\langle\left(\frac{\partial \log \mathcal{L}}{\partial p_{i}}\right)\left(\frac{\partial \log \mathcal{L}}{\partial p_{j}}\right)\right\rangle$,

which is the inverse of the covariance matrix depending on likelihood $\mathcal{L}$ and estimated parameters $p_{i}$. The expected value of this expression can be used to calculate how each data point contributes to the event parameters extracted from a fiducial model fitted to the light curve. The Fisher matrix simplifies in the $\chi^{2}$-case for a given time $t$ to:

$I_{i, j}(t)=\frac{1}{\sigma_{f}^{2}}\left(\frac{\partial f_{\text {model }}}{\partial p_{i}}\right)\left(\frac{\partial f_{\text {model }}}{\partial p_{j}}\right)$. 

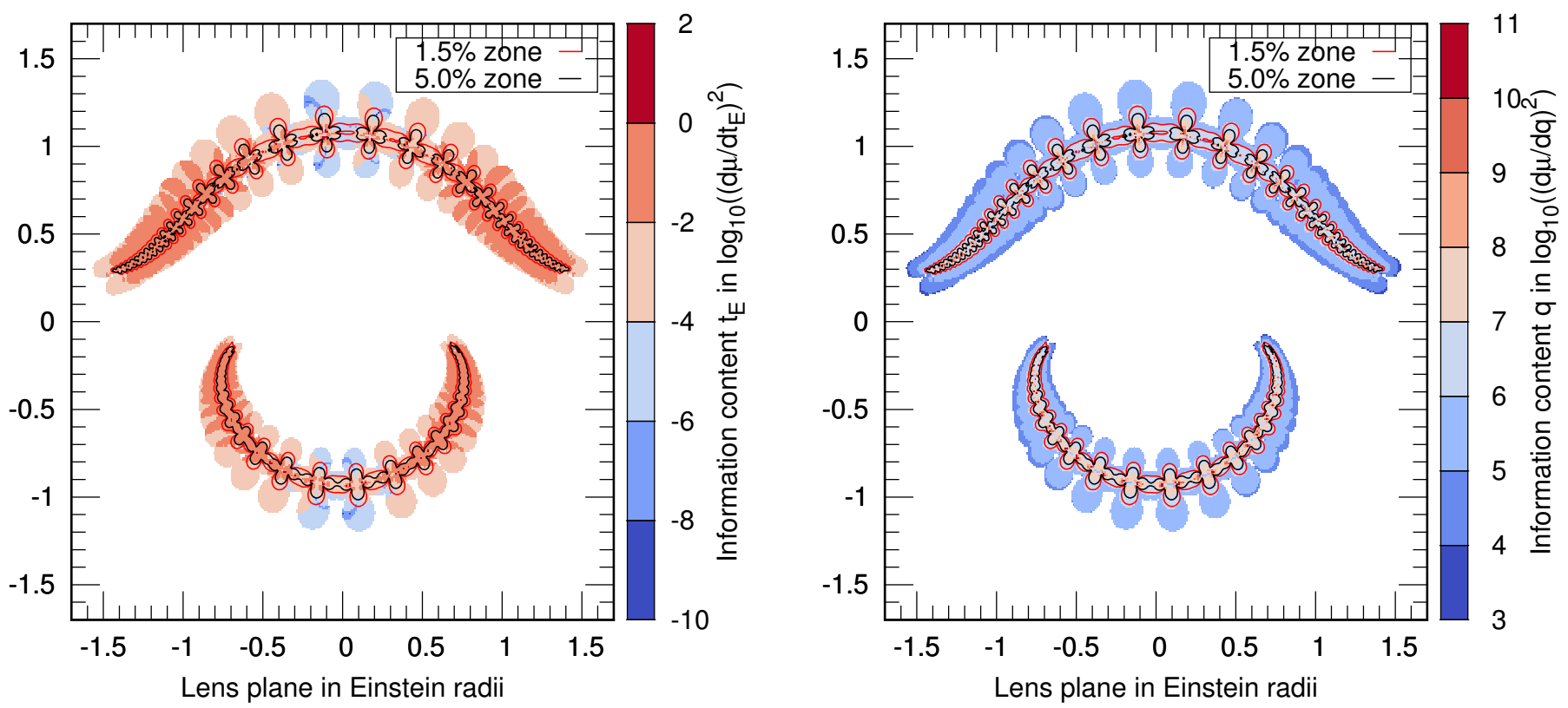

Fig. 6. Information content for characterizing $t_{\mathrm{E}}(l e f t)$ and $q($ right $)$ is shown for an unblended $q=10^{-4}$ light curve with known parameters. Each of the color-coded points corresponds to a potential planet and its maximum contribution for the given sampling (cf. Fig. 4). The mass ratio is optimally characterized by exactly following the detection zone area, while the Einstein time $t_{\mathrm{E}}$ could benefit from more observations in the wings. For $1 \%$ photometric accuracy, the color-coded logarithmic sensitivity just needs to exceed one in order to ensure $10 \%$ accuracy in each parameter. Since information is additive, one can estimate the number of required points.

Evaluating this expression requires only the best-fit model $f_{\text {model }}$ and the derivatives with respect to the parameters $p_{i}$. The information content of the full light curve is the sum of all matrices $I_{i, j}$. The microlensing model is usually given in the form shown in Eq. (2) and thus depends on $F(A(u(p)))$. Horne et al. (2009) and Dominik et al. (2010) focus on finding planets rather than characterizing parameters. Figure 6 shows areas associated with high probabilities of planet detection, for a typical detection threshold of $1.5 \%$ in photometric accuracy and mass ratios $q>10^{-4}$.

The sensitivity levels shown in Fig. 6 can be understood as inverse parameter uncertainties. In case of the mass ratio $q$, it follows directly the detection zone area, while for the Einstein time one needs a better coverage in the wings. The exact location of information maxima is not known before the peak and based on the distribution of these maxima, a sampling interval of at least $t_{\mathrm{E}} / 10$ is advisable. That holds for achieving about $10 \%$ accuracy in $q$, but there is an obvious caveat. The corresponding measurements need to be affected by the planet. For that purpose, our highest sampling interval is $15 \mathrm{~min}$ and follows $A^{1 / 2} t_{\mathrm{E}}$ (Horne et al. 2009; Dominik et al. 2010). We would also like to highlight that the azimuthal lobes in Fig. 4 overlap for the equidistant sampling. The radial detection zone lobes do not overlap to the same extent, which explains why the increased sampling rate at the peak is beneficial.

For low-mass planets we expect only a small perturbation in the light curve. Therefore, we estimate the contribution to the timescale based on a PSPL model. If the sampling rate aims to maximize our understanding of $t_{\mathrm{E}}$, the sampling strategy needs to be adjusted. The sensitivity with respect to a parameter $p$, such as the Einstein time $t_{\mathrm{E}}$, can be obtained from

$\frac{\partial F_{\mathrm{PSPL}}}{\partial t_{\mathrm{E}}}=F_{\mathrm{S}} \frac{\partial A}{\partial u} \frac{\partial u}{\partial t_{\mathrm{E}}}$ where

$\frac{\partial A}{\partial u}=\frac{-8}{\sqrt{u^{2}+4}\left(u^{4}+4 u^{2}\right)}$,

and the inner derivative is

$\frac{\partial u}{\partial t_{\mathrm{E}}}=\frac{-\left(t-t_{0}\right)^{2}}{t_{\mathrm{E}}^{3} u}$.

This implies that the sensitivity vanishes at $t=t_{0}$. The magnification $A$ can be approximated as $A \approx 1+\left(3 u^{2}\right)^{-1}$ (Horne et al. 2009), which leads to a simple estimate of sensitivity:

$\frac{\partial F_{\mathrm{PSPL}}}{\partial t_{\mathrm{E}}}=2 F_{\mathrm{S}} \frac{\left(t-t_{0}\right)^{2}}{3 t_{\mathrm{E}}^{3} u^{4}}$.

The latter expression has two implications for the sampling rate: to characterize $t_{\mathrm{E}}$ it should follow roughly $\left(t_{\mathrm{E}}^{3} u^{4}\right)^{-1}$ and the sampling interval $T$ should be scaled with $t_{\mathrm{E}}$. For the 2013 observing season, the requested sampling rate was kept independent of the Einstein time but, in $2014, t_{\mathrm{E}}$ was used to intensify the sampling of short events. By using the weighted average of the a priori variance $w_{0}$ from the $t_{\mathrm{E}}$ distribution of all events and the inverse variance from the PSPL-fit $w_{t_{\mathrm{E}}}=1 / \sigma_{t_{\mathrm{E}}}^{2}$, we ensure that the sampling rate is not adjusted before the peak when the parameter uncertainty in $t_{\mathrm{E}}$ is high:

$T \propto \sqrt{A} \frac{w_{t_{\mathrm{E}}} t_{\mathrm{E}} /\left\langle t_{\mathrm{E}}\right\rangle+w_{0}}{w_{t_{\mathrm{E}}}+w_{0}}$

\section{Implemented strategy in 2013 and 2014}

\subsection{Observing constraints: pre-selection}

In order to achieve a sensible pre-selection of events, we use empirical thresholds based on the achieved signal-to-noise ratio 
during the start of the pilot phase:

$t_{\mathrm{exp}} \approx \exp \left(0.995 I_{\mathrm{mag}}-11.042\right) \mathrm{s}$,

where the maximal exposure time was required to stay between 30 and $200 \mathrm{~s}$. The resulting expected accuracy has lead us to request events with a baseline $I_{\text {mag }}<18$ and a predicted peak $I_{\text {mag }}<17$. In addition, very long $t_{\mathrm{E}}>400 \mathrm{~d}$ events are excluded because they do not need to be observed at high cadence from follow-up observing teams. Most of the time we expect the telescope to be fully occupied with high priority targets and anomalies. If the event is well past the peak, non-anomalous events with $u>1.5$ are dropped from further consideration.

Independent of the constraints imposed by the system for automatic events, users can interact with the system through our online portal. As each site housed two or more telescopes, the target priority generator preferentially assigned events to telescopes that had already observed them. Additional observing resources on the $2 \mathrm{~m}$ Faulkes telescopes could be activated by the person responsible for the daily monitoring of operations. The exposure time and sampling rate for anomalous events was obtained from ARTEMiS (Dominik et al. 2008) and the SIGNALMEN anomaly detection algorithm, but exposure time estimates and sampling rates were adjusted for the $1 \mathrm{~m}$ network.

In order to assess how useful the suggested strategy is, we calculate the detection zone area as shown by Horne et al. (2009) and define the detection zone area as that area on the lens plane where a potential planet causes a deviation of at least $1.5 \%$ of magnification $A$. That implicitly assumes that we are able to adjust our exposure times accordingly. For that purpose, we use typical event parameters from $2012\left(u_{0}=0.15, t_{\mathrm{E}}=25 \mathrm{~d}\right)$ and introduce finite source effects as $\rho=10^{-3}$ Einstein radii for the binary model but not for the underlying point-lens model.

\subsection{Precedence for low-cadence fields}

Despite the increasing footprint of survey teams (see also Henderson et al. 2014), there are patches on the sky that cannot be visited more than once or twice per night. Therefore, we observe events more or less intensively, that is, events that are visited at least three times per night from OGLE are observed only once a night from the $1 \mathrm{~m}$ network to ensure early baseline coverage and reference frame. This rule only applies to regular events before reaching the peak and is our contribution to the microlensing community.

The priority flag requested by the network ${ }^{5}$ is set to low for events with $\Omega_{\mathrm{s}}<6$, to medium for $6<\Omega_{\mathrm{s}}<10$, and to high for $\Omega_{\mathrm{s}}>10$. The latter limit supersedes the nominal cadence by OGLE and ensures that all events can be observed on a regular basis. Events with $\Omega_{\mathrm{s}}<5$ are not queued at all. These limits do not refer to the current priority but to the maximum priority before uploading the target list. In the pilot phase of 2013, the estimates were made for a response time of 12 and later six hours. Since 2014, target lists are updated hourly. In addition to the $1 \mathrm{~m}$ telescopes, RoboNet was awarded observing time on a robotic $2 \mathrm{~m}$ telescope network consisting of the Faulkes Telescope North and South and the Liverpool Telescope. RoboTAP automatically requested $2 \mathrm{~m}$ data in case of $\Omega_{\mathrm{s}}>30$ for 2013 and for a manual selection of the events in 2014.

The target list for each telescope or for the telescope network is populated after estimating the total available observing time on the network. Anomalous events are added until $30 \%$ of the

\footnotetext{
5 See http://robonet.1 co.global/temp/tap1mlist.html
}

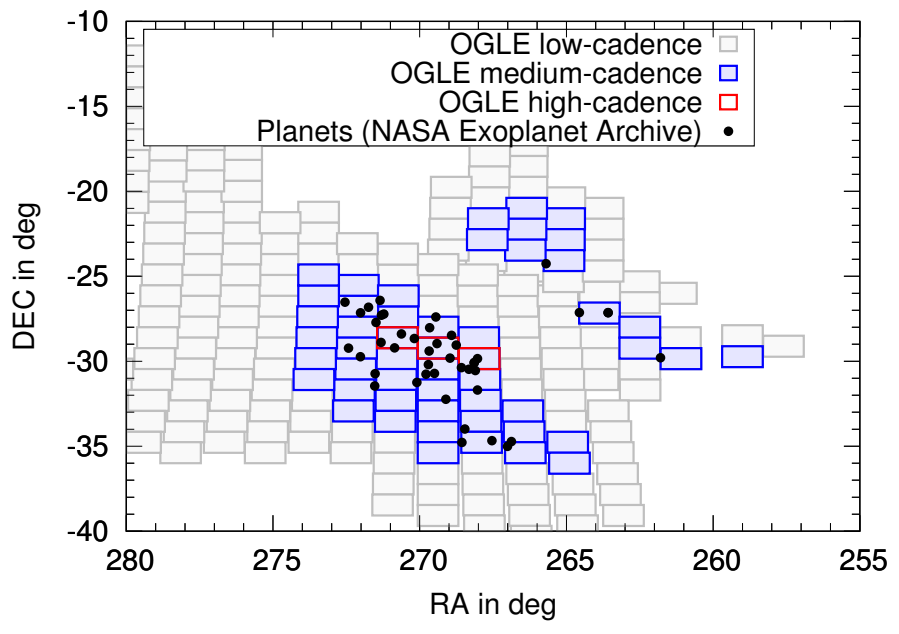

Fig. 7. Number and location of planets confirmed by 2017 (NASA Exoplanet Archive) is shown along with the survey cadence.

maximum available observing time is used up or one anomaly is requested per telescope. The latter can have a huge impact at the beginning and at the end of the season, where only a single anomalous event could be requested. The sampling interval is independent of anomalous events and is set on an event by event basis, partly automatically relying on the sampling rate recommended by ARTEMiS. At later stages team members can adjust the sampling interval depending on the event's exact shape of the light curve and the expected duration of the event. There are automatic and public fitting systems such as RTModel ${ }^{6}$ that help to assess the nature of the event, but at early stages the true nature remains uncertain.

The rest of the available time is populated with all other active events sorted by the aforementioned priority. In the pilot phase 2013 and at the beginning of the Key Project in 2014, there was no immediate targets of opportunity (ToO) system available. For extremely magnified events or likely planets, continuous observations of a single target were requested on a single telescope, redirecting all other events to the remaining telescopes on a given site. Since 2014 we can request urgent observations as target of opportunity, but due to the fast response of the scheduler (usually less than one hour) this is hardly ever necessary.

\section{RoboTAP in action}

RoboTAP generates target lists that are submitted to a target and observation manager code (ObsControl) that interfaces with the telescope network to request observations. In 2013 ObsControl read the telescope schedules directly per telescope and submitted microlensing observations into the gaps; once the network manager came online it just had to submit observation requests. The resulting requested observations were sent on a daily basis to the telescope network in 2013 and on an hourly basis in 2014 . As soon as observations have been taken, the system informs the team about its current state. For the Key Project the exact behavior of the scheduler changed slightly in the course of the project and no full-fleshed simulator of the scheduler was available. Instead, we have monitored how the scheduling worked in practice and if our desired target success rate was matched.

\footnotetext{
6 http://www. fisica.unisa.it/GravitationAstrophysics/
} RTModel.htm 


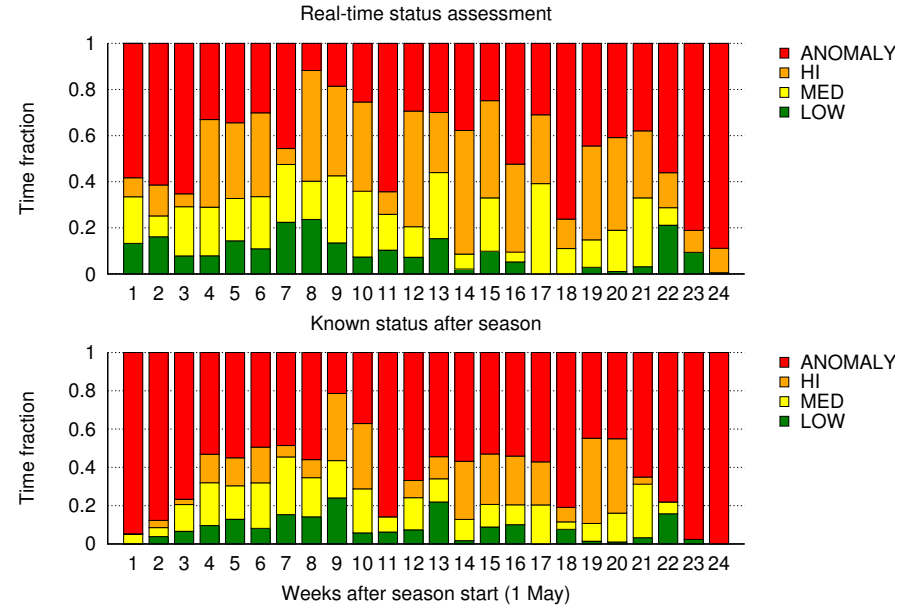

Fig. 8. Weekly fraction of events of each category based on the realtime assessment of the system (top panel) and the actual priority levels based on the parameters and the event status obtained at the end of the season.

The pilot phase season in 2013 lasted from 1 May until the end of September. During the season we logged the requested observing time by the target priority generator. Figure 8 displays a weekly chart of how the real-time priority knowledge compares to the known final state of the events at the end of the season. As expected, some events that have been identified as regular events turn out to be anomalous, and particularly those of high priority. The actual fraction of anomalous events, including manually alerted ones, differs slightly because single anomalous events at the beginning and end of the season can override the fraction of anomaly time we have envisaged. The agreement between the predicted priority level and the priority level for the final PSPL fit matches $82 \%$ of the requested observing time for regular events. For anomalous events, a successful coverage with either anomalous or high-priority status was achieved in $89 \%$ of observing time spent on anomalous events. These high success rates testify to the efficiency of the algorithm.

The field of view of follow-up telescopes constrains the number of targets that can be followed-up. In the microlensing pilot program up to 12 targets can be observed at the same time and on average seven events were on our target lists. Thresholds for rejecting events have been chosen in order to match the expected available observing time. The decision-making relied on PSPL fits using the SIGNALMEN anomaly detector (Dominik et al. 2007). For consistency, we have decided to focus our regular monitoring campaign on OGLE-IV ${ }^{7}$ events. The network approach enables us to follow more than one strategy and events from other survey teams are separately followed as targets of opportunity.

Figure 9 shows the time per week spent on microlensing targets. The change in overall shape between the two seasons can be explained by the deployment of the network scheduler distributing observing time between different projects. Nevertheless, the same overall pattern is apparent in both years, with the largest fraction of observing time allocated to anomalous and high priority events.

\footnotetext{
7 http://ogle.astrouw.edu.pl/ogle4/ews/ews.html
}

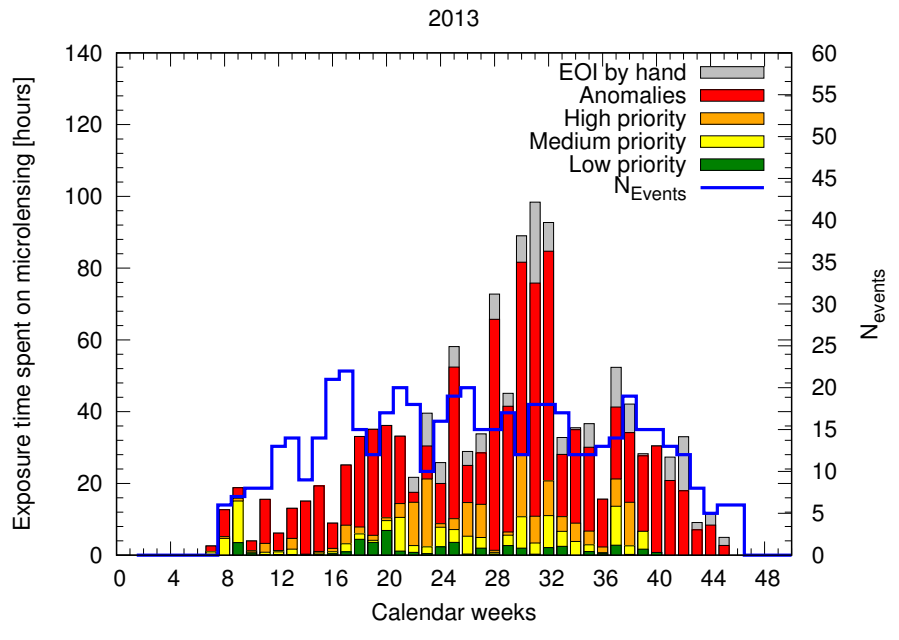

2014

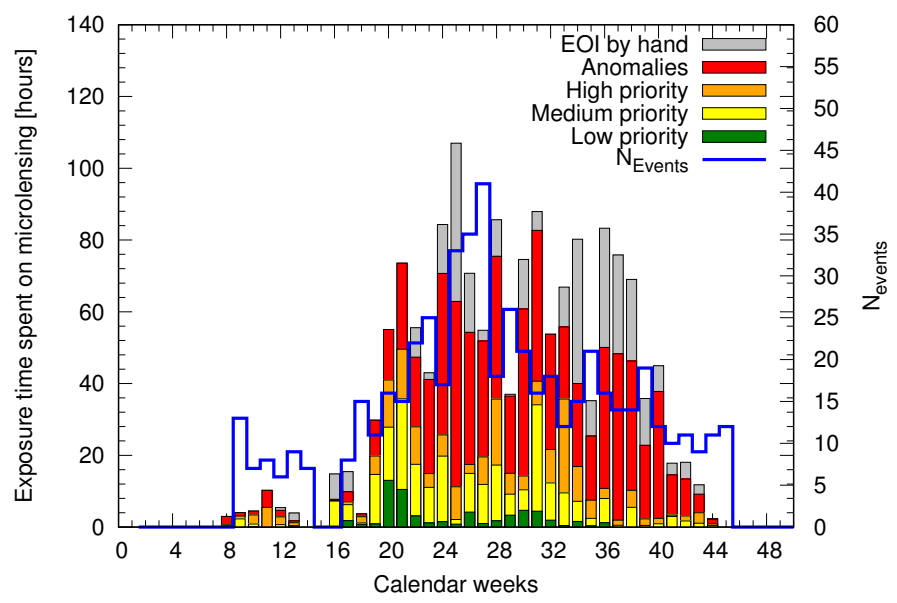

Fig. 9. Weekly observations with the LCO $1 \mathrm{~m}$ network of microlensing events in each category including manually requested observations, which are denoted as events of interest (EOI), are shown for the 2013 and the 2014 season. The continuous blue curve provides the number of followed events. The LCO scheduler came online after calendar week 18 in 2014.

\section{Expected number of planet detections}

To assess the number of planets that can be detected, we apply the following approach. Starting from the known PSPL model fit parameters for microlensing events in a given observing season, we employ the simplified Galactic model presented in Tsapras et al. (2016) to derive fiducial physical masses and circular orbital separations. The sample of planets drawn is calibrated to the orbital and mass distribution given in Cassan et al. (2012). Our results are still consistent when simulating orbits $a \in$ $[0.1,10] \mathrm{AU}$ and planet masses $M_{\mathrm{p}} \in[5,3178] M_{\oplus}$ distributed uniformly in $\log a, \log M_{\mathrm{p}}$.

Depending on the expected host star mass, we randomly assume that a multiple-star is present. The multiplicity fraction of stars and brown dwarfs is roughly approximated by a linear decrease in logarithmic mass from $80 \%$ for a $10 M_{\odot}$ star to $20 \%$ for a $0.1 M_{\odot}$ which is loosely motivated by what is known about stellar multiplicity (Lada 2006; Raghavan et al. 2010). For a uniform hourly sampling of events and based on the LCO exposure time calculator, for the longest useful exposure time of $300 \mathrm{~s}$ with the SBIG cameras we obtain $1.5 \%$ for average conditions ${ }^{8}$.

\footnotetext{
8 https://lco.global/files/etc/exposure_time_
} calculator.html 
ARTEMiS prediction success for $\Omega_{S}>6$ (medium priority)

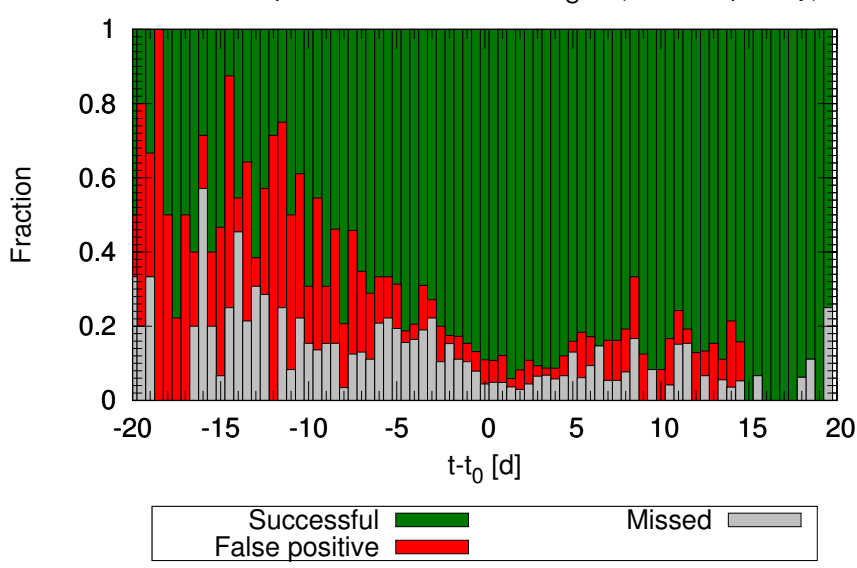

Fig. 10. Successful predictions, false positives, and missed predictions for PSPL parameters are shown as a stacked histogram, that is, the color-shaded areas correspond to the relative fractions.

The actual uncertainty can be larger than that and we can still detect a signal, but for uncertainties beyond $10 \%$ a planet can no longer be detected with the aforementioned criterion. As a side remark, we are exclusively focusing on planets orbiting singlestar hosts and we exclude circumbinary planets (Bennett et al. 2016).

Instead of simulating complete binary-lens light curves, we inject planets into a point-source point-lens model and test those seven points that have been observed closest to the anomaly to test if their cumulative $\Delta \chi^{2}>100$. In order to prevent single-outlier events, we request each observation to contribute by $\Delta \chi^{2}>100 / 7$. Requesting more points would exclude planets inducing only a short anomaly (Beaulieu et al. 2006), while discarding the threshold for each single observation would permit the detection of events that are only weakly varying and difficult to distinguish from systematic or long-term effects such as annual parallax.

If the expected annual sample of 2000 events could be used for our detections, we expect that 14 to 46 planets would be detectable orbiting single stars. The range of detectable planets corresponds to the $1 \sigma$ uncertainty of Cassan et al. (2012) and is used for all subsequent estimates. Only single-star hosts were considered and for that purpose $36 \%$ of all planets were discarded because they are orbiting potential multiple stars and a further $8 \%$ were discarded because their host mass was below $70 \mathrm{M}_{\text {Jupiter }}$. As a side remark, the observed fraction of identified binary star lenses is $2 \%$ (Tsapras et al. 2016) and that means that one third of our perceived single-lens events are actually multiple stars. Our most important idealization is an uninterrupted hourly sampling of all 2000 events with constant exposure time so that the SBIG cameras installed on the LCO 1m-network reach $1.5 \%$ photometric accuracy for an $I=18 \mathrm{mag}$ star. Our initial priority threshold for $\Omega_{\mathrm{S}}$ reduces the number of detected planets by $15 \%$ while reducing the requested time by a factor of 10 . The invested time still exceeds the scope of a Key Project by a factor of 50 .

All requirements together exceed the capabilities of existing observing teams. Estimating the yield for the anomaly-triggered part is more difficult. Our former estimates give a hint of the fraction of constituents. Based on $10 \%$ non-planetary anomalies and $2 \%$ of anomalous events, we can expect to find a planet whenever 50 events have been sufficiently covered.
We are still missing the contribution of the actual sampling strategy. For that purpose, we simulate light curves with the full range of sampling intervals and assign $60 \%$ of our allocated time for monitoring. So far we have assumed that the parameters of our light curves are appropriately prioritizing events at any given time. In practice, we are affected by uncertain event parameters. For a typical season, we determine the number of missed and incorrectly assigned priority categories as shown in Fig. 10. The estimates show us the event status at any given time compared to the final season parameter estimates. This leads us to a conservative estimate of $50 \%$ loss for events selected before the peak and roughly $20 \%$ after the peak. The expected planet yield decreases accordingly. In practice, the detection probability is higher in our selection of events and in the next section we will see how that supports our case.

\section{Achieved planet sensitivity}

From the observations taken in the seasons 2013 and 2014, we can estimate the expected number of planets from the nonanomalous part of our sample using the end of season pointsource point-lens parameter estimates. For the pilot phase 2013, we would have been sensitive to two to eight planets (for the reported light curve uncertainties), while the sensitivity in the regular observing season 2014 was increased to three to 11 planets neglecting the aforementioned fraction of missed and overrated events. Both estimates assume the Cassan et al. (2012) PMF and our seven-point detection criterion.

We start by simulating planets uniformly in $\log q, \log s$ where the separation $s \in[0.1,10]$ Einstein radii and $q \in\left[0.03,10^{-5}\right]$ (Bond et al. 2004). A planet is detected whenever the resulting $\Delta \chi^{2}$ exceeds 100 . Our reported photometric uncertainties are rescaled with one parameter so that $\chi_{\text {red }}^{2}=1$ because our light curves can come from up to eight telescopes. In addition, we keep the finite source radius constant for the binary model $\left(\rho=10^{-3}\right)$ and compare it with a PSPL model only. In a second step, we fit a point-lens model to the simulated planetary light curve and check if the detection can be smoothed out and the $\Delta \chi^{2}$ can fall below 100. As a side remark, we start the simulation using the ARTEMiS fit parameters, which include survey data. In the second step, we fit the model to follow-up data only. The detection is only accepted if it can be sustained by our followup data, but we do not claim that we can characterize the event without survey data. All accepted events are then divided by the number of samples per event and by that we obtain the expectation for the 118 regular events observed in the 2013-2014 season. Assuming each event contains one planet in the given range, we expect to find four in the season 2013 and five planets in the season 2014. Our earlier considerations are consistent as far as the expected planet yield is concerned. Defining an even more conservative threshold of $\Delta \chi^{2}>500$ as suggested by Yee et al. (2012) would reduce that by a factor of 1.4 , and thus to six planets for both seasons combined.

In order to obtain an immediate impression of how many planet candidates we have actually covered, we plot all events classified as planet candidates found on the RTModel web-page ${ }^{9}$ in Fig. 11. The number of covered events exceeds one planet per year, but half of the events were observed only after receiving an anomaly trigger, which also roughly matches the time allocation. The achieved planet detections nearly matches our expectation

\footnotetext{
9 http://www.fisica.unisa.it/GravitationAstrophysics/
} RTModel.htm 

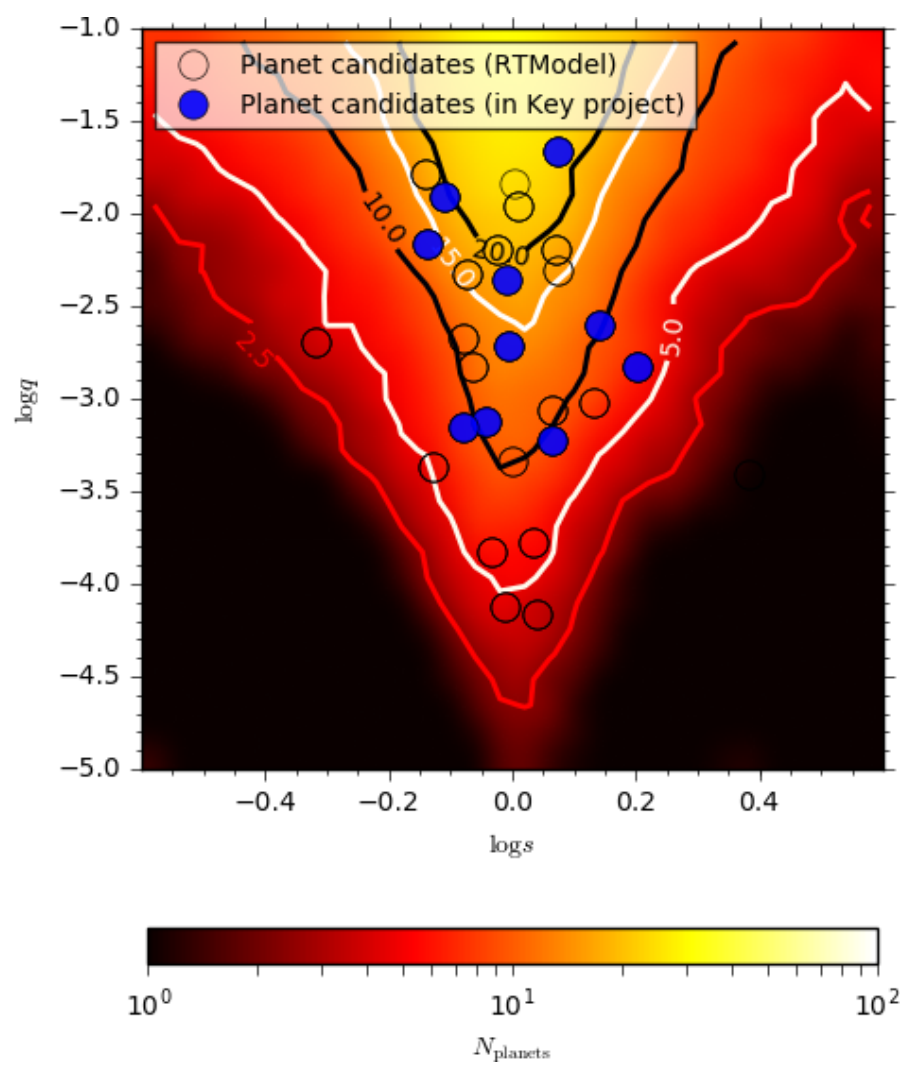

Fig. 11. Key Project sensitivity illustrated as expected number of detections assuming each event has a planet in the range $\log s \in[-1,1]$ Einstein radii and $\log q \in[\log 0.03,-5]$. To compare that with the number of detected planet candidates, we plot all planet candidates from real-time modeling efforts (RTModel) that have been reported in 2013 2014 as part of the Key Project (filled) and all other planet candidates that were reported in 2013-2016 (empty circles).

for the underlying regular event distribution and is consistent with Cassan et al. (2012).

In season 2015, a non-negligible fraction of observing time was spent on following Spitzer targets. This gives us the opportunity to see how a season with a partly different sampling strategy behaves. In order to observe all Spitzer targets, the observing cadence was gradually reduced in order to secure points on all ongoing events. In principle, that should double our planet sensitivity, but in practice we see a similar number of covered planet candidates and one event with crucial data for characterizing the event (Street et al. 2016). The unusually good coverage on that event also enables us to reach our target mass ratio of $10^{-4}$.

Independent of the exact sampling strategy required, we show in Fig. 12 how the actual assessment of the priority has changed over time and what the actual priority should have been based on the final parameter estimates. All targets shown in Fig. 12 have been reported online by the real-time modeling platform RTModel ${ }^{10}$ and observed by RoboNet. The priority thresholds discussed and chosen in this work are evidently allocating more observing time to events with lower magnification $(<50)$.

\section{Conclusions}

We have reported how follow-up observations of gravitational microlensing events can be automatically requested by our

\footnotetext{
10 http: //Www . fisica.unisa.it/GravitationAstrophysics/ RTModel/2015/RTModel.htm
}

Planetary candidates (RTModel 2015)
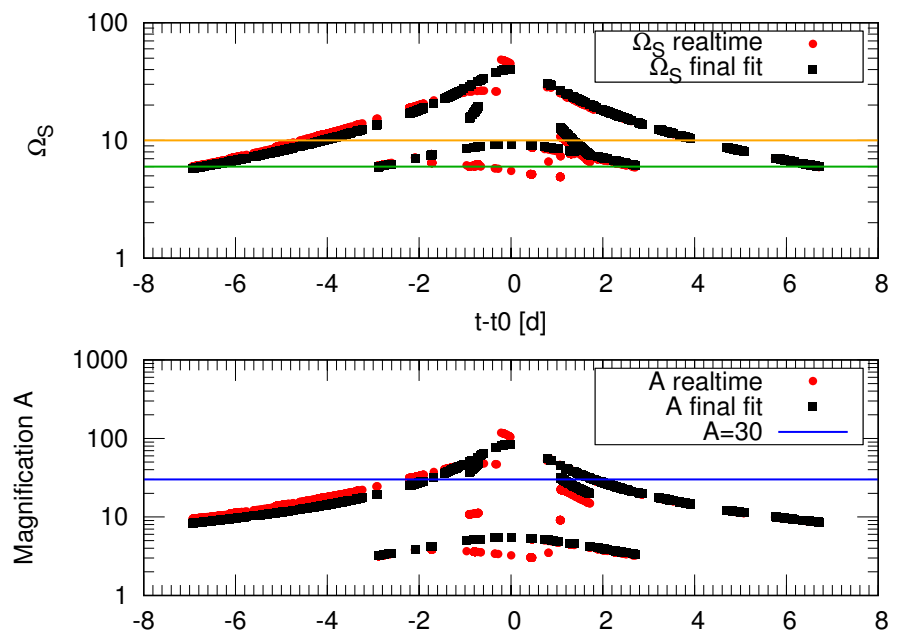

Fig. 12. Priority function and magnification is shown for those planet candidates that have been identified by RTModel (2015). High priority corresponds to a priority value above ten and means that the event is observed.

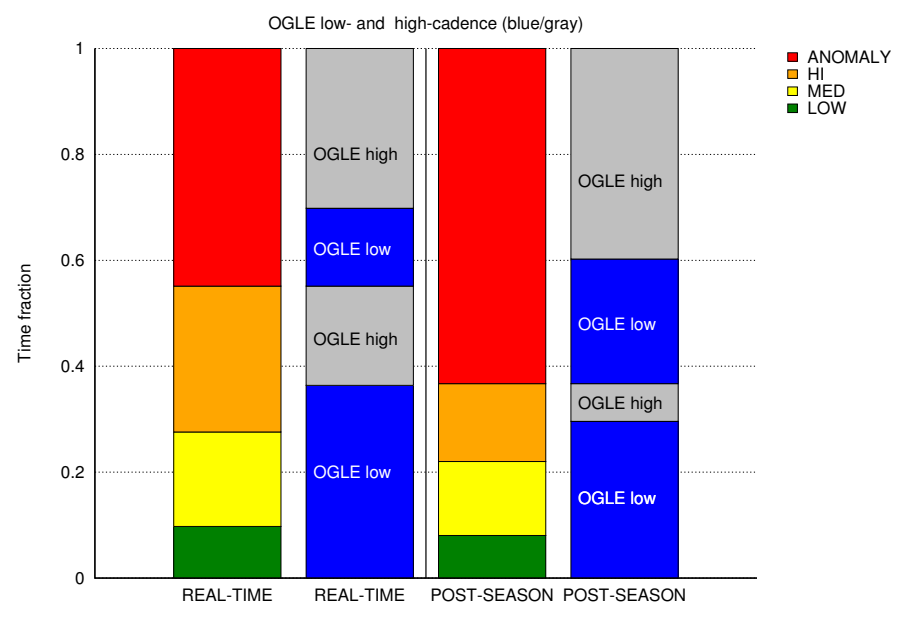

Fig. 13. Time fraction of different priority levels for regular and anomalous events based on the observing logs (left) and recalculated for parameters at the end of season (right). The figure highlights how many anomalous events have been identified as regular, high priority targets. It also suggests that events from high-cadence survey fields are more likely to be assessed as an anomalous event.

RoboTAP system. In that context, RoboTAP acts as an example of a broker between an incoming event stream and a robotic telescope network such as the LCO 1 m network. The 2013 pilot phase provided some valuable and well-tested insights into the way targets can be selected. One of the insights shown in Fig. 13 is our large contribution of observing time to help cover regular (PSPL-like) events in low-cadence fields, which are fields observed less than once per night by survey teams. Similar considerations will be required for all-sky surveys and their varying field cadence.

In the broader context of observing transient events triggered by future survey telescopes, like the Zwicky Transient Factory (ZTF) and the LSST, we have shown that respecting the survey cadence is very useful as a selector: it helps to avoid regions where surveys achieve sufficient coverage for the respective science goal. At the same time targets should not be selected when there is only little chance of triggering an anomaly in due time. 


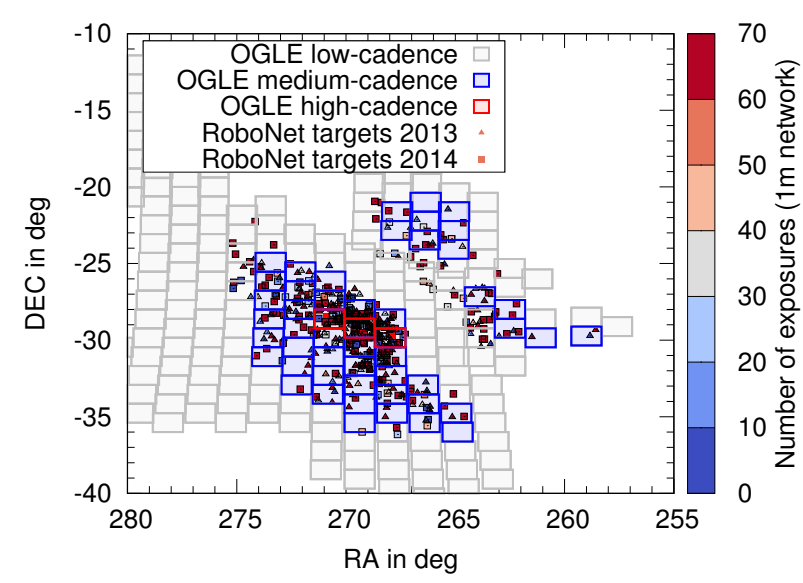

Fig. 14. Distribution of observed events and their number of visits during the RoboNet campaign 2013-2014 is shown along with high-, medium-, and low-coverage zones of the OGLE-IV survey.

In retrospect, $80 \%$ of our coverage went to regular targets. It is also noteworthy that regular events in the high-cadence zone in the real-time assessment more often turned out to be anomalous events and thus half of all observed events exhibited some form of anomaly.

Restricting follow-up observations to microlensing fields that survey teams can only visit once a night is not a viable option because event parameters and anomaly triggers remain elusive. For the fields defined in this work, the number of potential planetary events is reduced by a factor of 2 . Two factors contribute to that: larger event uncertainties, and thus inappropriately low or high priority levels, and the differing blend ratio. The former is also an indicator of the number of available source stars.

The anomaly detector requires multiple data points from two consecutive nights to deviate. Whenever surveys achieve only one to three visits per night, it is beneficial to follow anomalies from all fields. The overhead of low-cadence follow-up for the initial phase of the Key Project is shown in Fig. 14. Starting in 2015, we have changed the interpretation of what belongs to each cadence category. Only three fields with the very highest coverage by OGLE are subject to a reduced cadence during regular follow-up because we have concluded that OGLE and other survey teams will be able to characterize these targets by themselves.

Selecting microlensing events with high planet detection probability automatically ensures that the potentially limiting parameters for the planet mass $\left(q, t_{\mathrm{E}}\right)$ are sufficiently characterized. This is another way of expressing that some change of brightness that permits us to find a planet also contributes to the characterization of its parameters. However, a better coverage in the wings is the preferable choice for the Einstein time. For future observing programs we suggest to study in more detail of how to routinely include estimates of the expected achievable parallax signal in the selection process in a way that avoids a selection bias tending towards long duration events. Nevertheless, with current technology only half of the planets will benefit from that. In all other cases, we expect our approach to be appropriate.

When comparing the requested and observed fractions of all observing requests submitted to the telescope scheduler, we find that in $64 \%$ of all cases the requested next observation was either immediately after the requested sampling interval or at most 15 min later. That would be sufficient for detecting low-mass planets, but is not representative of present and future systems due to modifications in the target scheduling and a larger number of parallel projects. Our Key Project sensitivity suggests that low-mass planets with $q<10^{-4}$ should have been detected, but actual candidates are above a mass ratio of $q>10^{-3.5}$. Achieving a higher cadence, a higher photometric accuracy, and covering more events at the same time is usually hard to accomplish. The Spitzer campaign in 2015 was scientifically fruitful, not least because of the planet discovery of OGLE-2015-BLG0966 (Street et al. 2016). At the same time our nominal chance to detect planets was larger while the actual number of planet detections was reduced.

As far as finding and characterizing cool planets is concerned, we expected to detect approximately five planets per year. This estimate was consistent with the planet candidates identified in real-time modeling. Half of these candidates were identified in events with anomaly-triggered observations, while the other half were found in regularly monitored events. Further refinement of the results reported here will come from analyzing the data obtained by the next microlensing Key Project on the LCO network (2017-2020).

Acknowledgements. This publication was made possible by NPRP grant \# X-019-1-006 from the Qatar National Research Fund (a member of Qatar Foundation). Work by S.M. has been supported by the Strategic Priority Research Program "The Emergence of Cosmological Structures" of the Chinese Academy of Sciences Grant No. XDB09000000, and by the National Natural Science Foundation of China (NSFC) under grant numbers 11333003 and 11390372. Clément Ranc's research was supported by an appointment to the NASA Postdoctoral Program at the NASA Goddard Space Flight Center, administered by Universities Space Research Association under contract with NASA. K.H. acknowledges support from STFC grant ST/M001296/1. This work makes use of observations from the LCO network, which include three SUPAscopes owned by the University of St Andrews. The RoboNet program is an LCO Key Project using time allocations from the University of St Andrews, LCO and the University of Heidelberg together with time on the Liverpool Telescope through the Science and Technology Facilities Council (STFC), UK. This research has made use of the NASA Exoplanet Archive, which is operated by the California Institute of Technology, under contract with the National Aeronautics and Space Administration under the Exoplanet Exploration Program. We would like to thank the anonymous referee for his or her helpful comments and suggestions. Finally, we would like to thank all teams - and in particular the survey teams OGLE and MOA - for making their real-time data public.

\section{References}

Bachelet, E., Bramich, D. M., Han, C., et al. 2015, ApJ, 812, 136 Beaulieu, J.-P., Bennett, D. P., Fouqué, P., et al. 2006, Nature, 439, 437 Bellm, E. 2014, The Third Hot-wiring the Transient Universe Workshop, 27 Bennett, D. P., \& Rhie, S. H. 1996, ApJ, 472, 660

Bennett, D. P., Rhie, S. H., Udalski, A., et al. 2016, AJ, 152, 125

Bond, I. A., Udalski, A., Jaroszyński, M., et al. 2004, ApJ, 606, L155 Bozza V. 2010, MNRAS, 408, 2188

Bramich, D. M. 2008, MNRAS, 386, L77

Brown, T. M., Baliber, N., Bianco, F. B., et al. 2013, PASP, 125, 1031 Burgdorf, M. J., Bramich, D. M., Dominik, M., et al. 2007, Planet. Space Sci., 55,582

Cassan, A., Kubas, D., Beaulieu, J.-P., et al. 2012, Nature, 481, 167 Dominik, M. 2009, MNRAS, 393, 816

Dominik, M., Rattenbury, N. J., Allan, A., et al. 2007, MNRAS, 380, 792 Dominik, M., Horne, K., Allan, A., et al. 2008, Astron. Nachr., 329, 248 Dominik, M., Jørgensen, U. G., Rattenbury, N. J., et al. 2010, Astron. Nachr., 331,671

Gaudi, B. S., Albrow, M. D., An, J., et al. 2002, ApJ, 566, 463

Gould, A. 1992, ApJ, 392, 442

Gould, A. 2000, ApJ, 542, 785

Gould, A., \& Loeb, A. 1992, ApJ, 396, 104

Gould, A., Dong, S., Gaudi, B. S., et al. 2010, ApJ, 720, 1073

Han, C., Udalski, A., Choi, J.-Y., et al. 2013, ApJ, 762, L28

Henderson, C. B., Gaudi, B. S., Han, C., et al. 2014, ApJ, 794, 52

Horne, K., Snodgrass, C., \& Tsapras, Y. 2009, MNRAS, 396, 2087

Lada, C. J. 2006, ApJ, 640, L63

Law, N. M., Fors, O., Ratzloff, J., et al. 2015, PASP, 127, 234 
M. Hundertmark et al.: RoboTAP: Target priorities for robotic microlensing observations

LSST Science Collaboration, Abell, P. A., Allison, J., et al. 2009, ArXiv e-prints [arXiv: 0912.0201]

Mao, S., Smith, M. C., Woźniak, P., et al. 2002, MNRAS, 329, 349

Paczyński, B. 1986, ApJ, 304, 1

Park, B.-G., Jeon, Y.-B., Lee, C.-U., \& Han, C. 2006, ApJ, 643, 1233

Raghavan, D., McAlister, H. A., Henry, T. J., et al. 2010, ApJS, 190, 1

Ridgway, S. T., Matheson, T., Mighell, K. J., Olsen, K. A., \& Howell, S. B. 2014, ApJ, 796, 53

Saha, A., Wang, Z., Matheson, T., et al. 2016, Proc. SPIE, 9910, 99100F

Shvartzvald, Y., \& Maoz, D. 2012, MNRAS, 419, 3631

Smith, R. M., Dekany, R. G., Bebek, C., et al. 2014, Proc. SPIE, 9147, 914779

Snodgrass, C., Horne, K., \& Tsapras, Y. 2004, MNRAS, 351, 967

Snodgrass, C., Tsapras, Y., Street, R., et al. 2008, Manchester Microlensing Conference, 56

Steele, I. A., Smith, R. J., Rees, P. C., et al. 2004, Proc. SPIE, 5489, 679
Street, R. A., Tsapras, Y., Horne, K., et al. 2011, IAU Symp., 276, 459

Street, R. A., Choi, J.-Y., Tsapras, Y., et al. 2013, ApJ, 763, 67

Street, R. A., Udalski, A., Calchi Novati, S., et al. 2016, ApJ, 819, 93

Sumi, T. 2010, Pathways Towards Habitable Planets, 430, 225

Sumi, T., Kamiya, K., Bennett, D. P., et al. 2011, Nature, 473, 349

Tsapras, Y., Street, R., Horne, K., et al. 2009, Astron. Nachr., 330, 4

Tsapras, Y., Choi, J.-Y., Street, R. A., et al. 2014, ApJ, 782, 48

Tsapras, Y., Hundertmark, M., Wyrzykowski, Ł., et al. 2016, MNRAS, 457, 1320

Udalski, A., Szymanski, M., Kaluzny, J., et al. 1994, Acta Astron., 44, 227

Udalski, A., Szymański, M. K., \& Szymański, G. 2015, Acta Astron., 65, 1

Wambsganss, J. 1997, MNRAS, 284, 172

Wyrzykowski, Ł., Kostrzewa-Rutkowska, Z., Skowron, J., et al. 2016, MNRAS, 458, 3012

Yee, J. C., Shvartzvald, Y., Gal-Yam, A., et al. 2012, ApJ, 755, 102

Yee, J. C., Gould, A., Beichman, C., et al. 2015, ApJ, 810, 155 